\title{
A complementing approach for identifying ethical issues in care robotics - grounding ethics in practical use
}

\author{
Stina Nylander, Sara Ljungblad, Javier Jiménez Villareal
}

\begin{abstract}
We use a long-term study of a robotic eating-aid for disabled users to illustrate how empirical use give rise to a set of ethical issues that might be overlooked in ethic discussions based on theoretical extrapolation of the current state-of-the-art in robotics. This approach provides an important complement to the existing robot ethics by revealing new issues as well as providing actionable guidance for current and future robot design. We discuss our material in relation to the literature on robot ethics, specifically the risk of robots performing care taking tasks and thus causing increased isolation for care recipients. Our data identifies a different set of ethical issues such as independence, privacy, and identity where robotics, if carefully designed and developed, can make positive contributions.
\end{abstract}

\section{INTRODUCTION}

Robots are becoming increasingly ubiquitous and the field is currently under rapid development. On one hand this is highly appreciated since they replace humans in performing tedious, repetitive, and even dangerous tasks, and on the other hand they are criticized for leaving human workers without jobs and undermining their competence. This tension illustrates the complexity of our attitudes, understanding of, and relation to robots, partly originating from influences from fiction. Books such as Asimov's I, Robot, movies such as the Star Wars series, Terminator, and RoboCop, and computer games like Portal and Mass Effect, paint vivid portraits of highly skilled robots that sometimes cannot be distinguished from humans. In addition, the most common theme in robot fiction being robots taking over the world, suppressing humans, thus ending our way of life as we know it creates an underlying skepticism and fear that shapes our attitudes toward robots (Ferneus et al., 2009).

Even though robots for home use such as vacuum robots (Roomba, NaviBot) or robot pets (Pleo, AIBO) are becoming more common place, most people have little practical experience of interacting with robots in everyday life. Thus they have little real-life experience to balance the strong projection from fiction. This makes it hard for most people to understand what robots can do in reality, what is the realistic

Research supported by the LIREC project under contract FP7-215554.

S. Nylander is with Swedish Institute of Computer Science, Box 1263, 16429, Kista, Sweden (phone: +468 6331500, fax +46 87517230 , e-mail: stny@sics.se).

S. Ljungblad is with Lots design, Kastellgatan 1, 41307 Göteborg, Sweden (e-mail: saral@ sics.se).

J. Jiménez Villareal is with Royal Institute of Technology (KTH), SE10044, Stockholm, Sweden (e-mail: javier.jimenez.villareal@ gmail.com). state-of-the-art of robot performance, and what is a realistic time frame for approaching certain functionality.

Therefore, many ethical discussions around robotics and how we will, or should, use robots in the future are not based on users' experience of robots, or how useful they find them, but on theoretical discussions extrapolating on how robots should or could be used, and what the implications of that would be. It is important for the robot community to look forward and try to anticipate both functional and ethical issues of new technologies, but without empirical grounding it is easy to overlook the effect of users appropriating the technology as well as to overestimate the speed of technical progress in such discussions. Moreover, conclusions from discussions based on extrapolation from a current situation to a distant future do not provide actionable guidance for design and development of robots and robotic artifacts at present and in the near future.

History shows that it is difficult to predict how new technology will develop and what effects that will have. This is well illustrated by other technologies that were ground breaking when introduced and that have proven to have quite different impact on society and people's behavior than what was initially expected or even feared. When the telephone was introduced in the US it was said that people would stop visiting their friends and only talk to them on the phone (Fischer, 1993). Reality has shown that this was not the case. We are talking more than ever to our loved ones in our cell phones, and at the same time travelling more than ever. When vacuum cleaners were introduced in our homes the general belief was that they, and other cleaning technology, drastically would reduce the amount of time used for cleaning our homes. What really happened was that the level of what was considered a clean home changed. We spend the same amount of time cleaning with the new tools, and have much cleaner homes today (Cowan, 1983). This does not mean that telephone and vacuum cleaners are problem free technologies, just that we could not predict their social impact. Possibly, the case of robots is similar.

We certainly acknowledge the difficulties with grounding ethical discussions about new technology or technology in rapid progress in practical use, and we are definitely not arguing for it replacing the theoretical discussion. However, here we want to point out that it is possible to gather practical data to inform ethical discussions, and describe an example that illustrate how such practical data can illustrate different ethical issues than those commonly pointed out in foresight 
papers. This could on the one hand complement the theoretical discussion with otherwise neglected ethical issues, and on the other hand it would provide the robot research and development with concrete and actionable input on how to go forward in a short-term perspective.

We will use the area of robots for care as our example, drawing upon the large literature on the danger of isolation should we start using robots on a large scale for care. The discussion will be grounded in our experience from a longterm user study of a robotic eating-aid that uncovered a set of ethical issues rather different from those usually treated in the discussion about robots in care and the associated risks of isolation.

\section{ROBOTS AS CARERS}

A common theme in the discussion about future robots is elderly care. Many countries face an ageing population where fewer young are available to care for the elderly ((SCB, 1999), (WHO, 2007)). Several countries, perhaps with Japan and Germany at the forefront, have turned to robotics as one solution to this problem.

In elderly care, there are three broad areas wherein robots are expected to be useful: assist elderly and/or carers in their tasks, monitor health and behavior, provide companionship (Sharkey \& Sharkey, 2010a).

Assistance can be provided in many different ways and by accordingly different robots. Tasks can be simple and limited, performed by specialized robots such as vacuuming robots like the Roomba or NaviBot or eating-aids such as Bestic or MySpoon that assist users in feeding themselves. More complicated tasks such as personal hygiene would require more advances robots.

Health and behavior monitoring Robots in people's homes could record user behavior relevant to health and safety, such as a person not getting out of bed, falling and not being able to get up, or staying too long in the bathroom. In such situations robots could alert health services. Other types of behavior such as eating habits or medication could be also monitored and relayed to physicians or family members.

Companionship Robot companions do not perform any assistive tasks but are designed to provide company, distraction, and to be social and interactive in general. This type of robots has been commercially available for more than a decade in the form of robotic pets such as the Sony AIBO robot dog, the Pleo dinosaur, and the Paro Seal.

A less discussed, but still relevant, topic for discussion in relation to robot carers is child care (Sharkey \& Sharkey, 2010b, Borenstein \& Pearson, 2012).

\section{ROBOT CARERS AND ISOLATION}

An often voiced caution on the topic of future use of robots in care is that robot carers would increase the isolation among the elderly.
Sparrow \& Sparrow (2006) and Borenstein \& Pearson (2012) believe that robots in care will replace human carers. They argue that if future robots can perform higher level tasks in care taking they definitely will replace human carers for economic reasons. Even if robots would be used for simple, seemingly non-social tasks to assist human carers it would have effects on the social situation for elders. The role of caring for elders often is tightly coupled with household tasks such as cleaning. If robots take over some of those tasks, human carers will have fewer reasons to come, and they argue that some of the few opportunities elders have for human contact will disappear since cleaning staff will be replaced by robots. They find it naïve to think that successful robots will only be used in combination with, and as support, for human carers. If robots will be able to perform tasks in elderly care they will contribute to a reduction of the number of human carers, and thus increase the isolation among elders.

Sharkey \& Sharkey argue that using robots to assist human carers with tasks such as lifting, carrying or cleaning might reduce the amount of human contact (Sharkey \& Sharkey, 2010a). They argue that daily, simple care tasks such as changing nappies, adjusting clothes etc. are important for forming relationships, and letting robots do this would deprive care recipients from important human contact. If robot carers took care of daily routines, elders would risk being left alone for long periods of time. An extreme case of this would be children left alone for long stretches of time with robot nannies. Since it is unlikely that robots in any foreseeable future can mimic the cognitive capabilities of humans, leaving children in the care of robots could possibly cause psychological problems (Sharkey \& Sharkey, 2010b). Using robots for surveillance and sensing could also reduce human contact, since increased surveillance could make carers and family members less inclined to visit elders to check up on them (Sharkey \& Sharkey, 2012).

Parks (2010) notes that there is already isolation among elders living both at home and in nursing homes, but believes that robots would make things worse. The existing isolation is one reason for elders resisting the use of robots. Visits by human carers are so important that many elders prefer their help with showering and other personal hygiene even though this is something many people would prefer not having another person do for them. However, this is often the only social contact and human touch available to them.

We believe that the issue is more complicated than robot carers replacing human carers and thus causing increased isolation for care recipients. It is possible that robot carers also could help care recipients become more independent and create a way of life less dependent on human carers (Borenstein \& Pearson, 2010). Furthermore, the risk of isolation as it is described in the literature provides little guidance about how to create robotic products that does not cause increased isolation. The area of assistive technology does not provide advanced robot carers but offer real-life use of robotic technology which we believe is important input to 
the discussion on robot ethics. Below, we illustrate how an approach grounded in actual use of a robotic product, the eating-aid Bestic, can reveal other aspects of robots in care than the extrapolation from today into a distant future, and thus provide a complementing perspective on the ethics of robot carers.

\section{The Bestic EXAMPle}

'Bestic' is a robotic eating aid, supporting people who cannot feed themselves due to disabilities in hands and arms. Bestic is designed to look like a kitchen aid, is equipped with a robotic arm that has a spoon attached, and is programmed to simplify the action of picking up food from the plate. The user can steer the product with different operating devices adapted to their disability. The user decides which area of the plate should collect the food from by steering the spoon and presses a button to automatically collect the food from that area. Normally, someone else has to chop the food, serve it and collect pieces that may accidentally be pushed outside the plate. Bestic is not designed to support any other user activities, and is thus only intended to support the act of feeding oneself.

Although limited in its functionality, we believe that a study of long term use of Bestic can shed important light on ethical issues around the use and experience of an assistive robot in daily life. We therefore conducted several interviews each with four users of Bestic (Jiménez Villareal \& Ljungblad, 2011). Even though it was under evaluation and redevelopment during our data collection, the situated use over time gave users the ability to reflect on what an eatingaid would do for them and how that would implicate their lives.

There are products on the market that are similar to Bestic, such as the Meal Time Partner (http://mealtimepartners.com/), My Spoon (http://www.secom.co.jp/english/myspoon/), and the Winsford Feeder (Patterson medical). We have used Bestic for this study since the company that manufactures it wanted our input for their development process.

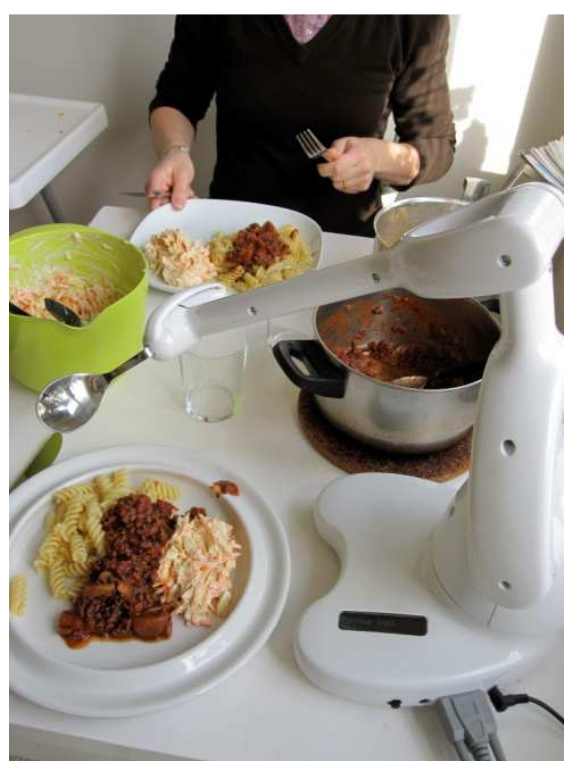

Figure 1. About to eat lunch, with Bestic.

\section{A. Data-collection}

The interviews with users of the eating-aid were carried out in their home environment, often by sharing a meal with the interviewees. Several weeks or months passed between interview sessions. The interviews were qualitative in nature and lasted for about an hour.

'Carl' is 37 years old and has Cerebral palsy, which results in spasticity (involuntary movements and tightness of muscles). He is assisted by an electric wheelchair (a 'Permobil') and personal assistants. Carl was very optimistic from the start, and looked forward to eat without an assistant. However, he ended up only using the system for two weeks, because his involuntary movements made it uncomfortable and difficult to eat with the system.

'Erik' is 10 years old and has a lack of movement capability in his arms. He has now used Bestic for 10 months. He uses Bestic daily or every week, and is satisfied with the system, except for when eating soup, when the system spills too much. He has expressed the view that it is more fun to eat with Bestic, because you can choose and you don't have to say what you want (compared to being fed).

'Heidi' is 70 years old and has an autoimmunity disease, which leads to an immune response against her own cells and tissues. She uses a Permobil and cannot lift her arms. She used the system during two different periods. She used one early version for four months and then a second generation of Bestic for ten months. She stopped using the system because she was spilling too much.

\section{PRIVACY, INDEPENDENCE, AND IDENTITY}

Our analysis has revealed interesting findings on privacy, independence, and identity connected to the use of Bestic. 


\section{A. Privacy}

Carl, one of the Bestic users, who is 35 years old and is usually fed by an assistant, explains his experience:

"It is directly more pleasant to feed yourself than to have an assistant." His wife describes: - It's much more private, you can talk about whatever you want.

Carl about assistants: "They are a bit uncomfortable when they feed me. I'm thinking of what may cause that. It's an intimate situation."

This shows how the Bestic eating-aid helps to create a more private eating situation since Carl can have a meal with his wife without having an assistant present and not have his wife feed him. Bestic allows them both to have a social experience since they both feed themselves and no one has to help the other. They can talk to each other without having Carl's assistant listening and they do not have to include the assistant in the social conversation. Bestic is a specialized tool and will not replace Carl's assistant, or create a situation where he never needs help from his wife, but it creates a more private and independent eating situation.

\section{B. Independence}

For Erik, the ten-year-old who has been using Bestic for several months, the robot provides independence in the eating situation. When using Bestic he can choose at what pace he is taking each spoonful of food, and what type of food he wants to have on the next spoon. To him, this independence is important and compensates for the fact that he spills a lot of food when eating with Bestic.

Erik about being fed by someone: "It is not so much fun. It is awkward too, when I'm fed because then I need to tell when I want more."

As for Carl, Bestic gives Erik the opportunity to have a meal with his family on the same terms as everyone else. He can feed himself without any help and can participate in the conversation without switching between giving directions about how he wants to be fed and social interaction. Erik does not have an assistant since he lives with his parents and his disability is not as severe as for example Carl's. However, he of course needs help with various activities from his family. Again, Bestic will not make him independent in every situation and thus replace his family members but in the specific situation of eating it gives him independence and control of his experience.

\section{Identity and self esteem} Bestic.

Heidi highly appreciated the design and form factor of

"It is small and cute ... and I really like the way it looks when it stretches its arms above its head with the food."

The design of assistive technology is highly important. The advantages that technology can offer in terms of for example privacy and independence can easily be outweighed by a design that projects disability. Our participants were clear on the fact that they did not want technology that drew attention to their disability, or in other ways pointed out that they were different. The design of Bestic evokes kitchen appliances such as blenders or bread mixers rather than assistive technology. This made our participants willing to use Bestic in social situations where other people were present, mostly due to the aesthetics of the device.

Interestingly enough it was another aesthetical aspect of Bestic that made Heidi stop using it. It dropped so much food on her clothes that she did not feel comfortable. She said she looked like a baby after a meal, and she did not like that at all.

"Bestic drops food so I feel like a two-year-old with food everywhere, when I eat soup I need towels on the table and on myself."

She would never use Bestic in a restaurant for this reason either. The eating situation is about so much more than simple sustenance. It is a social situation which we want to share with friends and family, and something that appeals to our senses. It is about taste and look, and about enjoyment. For Heidi, the joy and pleasure of eating was limited due to the food spilling.

\section{WHAT DOES GROUNDED ETHICS OFFER?}

We do not in any way claim that robot ethics discussions should focus solely on existing robots and their use and stop looking forward and try to anticipate what could happen in the future. To continue the discussion of isolation, it is a real problem that needs to be dealt with in many ways. It is not a new problem though, and not a problem that only occurs in relation to robots in care. Parks ( 2010) states that many elders already are lonely regardless if they are living at home or in nursing homes, and an important source of social contact are the people who help them with personal hygiene and practical matters such as cleaning. Using robots for some of these tasks could very well mean a reduction in human contact for already lonely people.

However, what we would like to argue for is that extrapolating from the present situation to a distant future will not give the full picture of ethical issues around robots in care. The claims that robots in care would increase isolation among elders presented for example by Sparrow \& Sparrow (2006), Sharkey \& Sharkey (2010a), and Parks (2010) are not based on trends in actual use of robots. Rather, they are extrapolated from promises, hopes, or expectations from manufacturers, researchers and the like, and contain rather important simplifications and assumptions that the development will go straight in one direction from here. The leap from the present situation to robot carers is substantial since there are few examples of robots actually replacing human carers other that in small experimental or research settings, which is also noted by Sparrow \& Sparrow (2006). Moreover, they apply a perspective of cause and effect that is quite simple and exclude many factors that influence people's choices in general and uptake of technology in 
particular. Factors such as sociality, emotion, and aesthetics are less discussed even though they are strong motivating factors in people's lives.

When looking at real examples, the trend points in a rather different direction than robots replacing human carers. At present, existing robots are fit to perform specialized tasks in a restricted space. We argue that a robotic product can support a very specific need, where a personal assistant is not necessarily part of the desired experience, thus addressing successfully the need and user's preferences without eliminating the need for an assistant. Bestic and its peers assist users in the isolated task of feeding themselves when the food is cooked and served. Human assistants are required to provide necessary ingredients, cook, serve, and cut the food, before Bestic or MySpoon can be used at all. After the meal, human assistants need to clean the dishes as well as the Bestic robot. In the same way, vacuuming robots perform the isolated cleaning task of vacuuming. They cannot do the preparation needed such as clearing the floor from obstructing things, and they perform none of the other cleaning tasks required in a home such as washing up dishes, cleaning toilets, changing bed sheets, doing laundry, or get more detergent and toilet paper from the store. It has been shown that vacuum robots not necessary save time since the space need to be prepared for them and they need to be moved between floors, cleaned after use etc. (Sung et al., 2007, Forlizzi \& DiSalvo, 2006). From these examples, it seems rather farfetched that robots will replace human carers or human cleaners in any near future.

This, however, does not mean that it is pointless to study existing robots from an ethical perspective. We argue that this could be extremely useful for the future of the field, since such grounded ethics can provide concrete and actionable input to the design and development of next generation of robots for home use, thus complementing the ethics of extrapolation that spans a longer stretch of time and therefore is quite general in its advice. The example of Bestic shows several important ethical issues that could guide the design and development process of assistive robots.

First, our study shows that an eating-aid like Bestic provides independence and freedom to the users in the eating situation. Second, it puts the social function of having a meal in focus since everyone present can feed themselves in an equal way. Third, the physical design and framing of assistive robots have substantial importance for the user experience, the willingness to use them, and the social impact of needing assistance.

These issues offer practical guidance to the robotics field and will be further elaborated on in the following section.

\section{DESIGN IMPLICATIONS}

It is not unknown that assistive technology can provide independence (Borenstein \& Pearson, 2010), but we believe that it is relevant to revisit this area in the light of ethics and our empirical data. Bestic illustrates that even independence within very narrow frames can dramatically impact users experience of a device as well as a social situation which makes it worth striving for in design. Bestic provided our participants the freedom to feed themselves according to their own preferences and feelings, without having to give directions to a human assistant about how they want to eat, what they want next, and the pace they want to eat in. This is an extremely limited independence but still had important impact on the social situation as well as how they experienced a frequent and mundane situation of having a meal. Similarly, the use of vacuum robots might allow elders to choose when to vacuum, even though they need help with other parts of the cleaning process including necessary preparations for the robot. The value of independence and freedom even within limited situations highlight the importance of user control. Specialized robots might have limited capabilities but can still enforce the users' control over their own lives. Designing robots that promote such user control, and independence in such limited situations would provide value to users without significantly increasing the risks for isolation due to elimination of human carers. Important challenges for design to promote such user control are for example interaction techniques suitable for assistive robots. The concept of ActDresses provide an interesting example on how users can interact with robots and control their behavior by accessories and clothing (Jacobsson et al., 2010, Ferneus \& Jacobsson, 2009).

Isolation is not the only potential social impact of assistive robots. Our material shows that an eating-aid like Bestic can play an important role in creating a social situation around the activity of eating, and even increase privacy. Taking the focus away from helping the person who cannot feed themselves, and eliminating the need for a human assistant during the actual meal, helped making the eating situation pleasant and private. Thus, by providing certain independence assistive robots could also make life more social. Robots do not necessarily need social skills, empathy, or emotion to support the social life of their users. In the case of Carl, Bestic created increased privacy by allowing Carl to feed himself instead of being fed by a human assistant. We believe these social benefits of Bestic illustrate that robotic products have the potential to introduce positive elements in the care of elders and disabled. We should not stop our ethical reflection at the risk of isolation that comes with robot carers, but delve deeper into the details of the care situation, the social context, and the interaction with robots. Robot carers will shape the future care, however, we must not forget that these robots will be shaped by the care situation and the people involved. As with the telephone, social and practical elements will have high impact on the future results. Here, grounded ethics can be a valuable link between ethics, design, development, and use.

Finally, aesthetics was revealed as a highly important factor for the use and perception of Bestic. The design of future assistive robots need to consider the fact that robots 
for home use need to fit people's homes and aesthetics (Sung et al., 2009). In the case of personal robots for assistance they might be brought to places and situations outside the home which brings personal aesthetics and image management into the picture. Users of assistive robots might not necessarily want to project an image of being disabled and the design of robots need to take this into account. Here, personalization might play an important role. Support for personalizing assistive robots would give users means for projecting their own personality and values through the assistive technology, rather than being shaped by it. Designers and developers of robots can play an important role in creating tools and support for personalization (Sung et al., 2009) for future assistive robots.

\section{FRAMING}

A grounded perspective on ethics also keeps the discussion focused within a scenario that is easier to understand for intended users. Given the capabilities of current assistive robots, a grounded discussion will not talk about robots as autonomous people that can do everything we would like them to as they are portrayed in movies. If we talk about robots as tools, such as knives and forks, vacuum cleaners and washing machines, we end up with different ethical issues than if we talk about robots as people, assistants, or nurses. A grounded discussion would not call Bestic a robot assistant or a robot carer since its capabilities are very limited (Ljungblad et al., 2011). Instead, framing Bestic as an eating-aid invokes realistic expectations of its capabilities and thus also of its consequences. A similar case is RobCab, a hospital robot purposely framed as a transport robot and not as a robot nurse to invoke realistic expectations among hospital staff, and reduce fear of being replaced by a robot (Ljungblad et al., 2012).

We believe that framing robots and robotic products in real-life use and real-life settings help developers, potential users, as well as researchers to create images of robots that balance the ones from fiction. This will shed important light on user needs, ethical issues, and design challenges within the field of robotics.

\section{CONCLUSION}

We have described above how a small empirical study of a specialized robotic product can reveal a set of ethical issues that are important for its future users. Such ethical issues provide guidance for robotic design and development on how to proceed and what to strive for in current and future work. It is our firm belief that the strength of the work presented here lies in the connection to real-life use. Even though Bestic has very specialized functionality and our study was small, the situated use over time still revealed important issues. Larger studies of robotic products would certainly yield even more ethical input to the design and development of future robots.

\section{ACKNOWLEDGMENT}

We would like to thank our participants, their families, and their assistants, for their input to this work, as well as the Bestic development team for helping and support. This work was conducted within the LIREC project funded by the European Commission FP7-ICT under contract FP7-215554.

\section{REFERENCES}

[1] BORENSTEIN, J. \& PEARSON, Y. (2010) Robot caregivers: harbingers of expanded freedom for all? Ethics and Information Technology, 2010, 277-288.

[2] BORENSTEIN, J. \& PEARSON, Y. (2012) Robot Caregivers: Ethical Issues across the Human Lifespan. IN LIN, P., ABNEY, K. \& BEKEY, G. A. (Eds.) Robot Ethics. MIT Press.

[3] COWAN, R. (1983) More Work for Mother, Basic Books.

[4] FERNEUS, Y. \& JACOBSSON, M. (2009) Comics, Robots, Fashion and Programming: outlining the concept of actDresses. TEI.

[5] FERNEUS, Y., JACOBSSON, M., LJUNGBLAD, S. \& HOLMQUIST, L. E. (2009) Are We Living in a Robot Cargo Cult? HRI. ACM Press.

[6] FISCHER, C. S. (1993) America Calling, University of California Press.

[7] FORLIZZI, J. \& DISALVO, C. (2006) Service Robots in the Domestic Environment: A Study of the Roomba Vacuum in the Home. HRI.

[8] JACOBSSON, M., FERNEUS, Y. \& TIEBEN, R. (2010) The Look, the Feel and the Action: Making Sets of ActDresses for Robotic Movement. DIS.

[9] JIMÉNEZ VILLAREAL, J. \& LJUNGBLAD, S. (2011) Experience Centred Design for a Robotic Eating Aid. HRI, Poster. ACM Press.

[10] LJUNGBLAD, S., KOTRBOVA, J., JACOBSSON, M., CRAMER, H. \& NIECHWIADOWICZ, K. (2012) Hospital Robot at Work: Something Alien or an Intelligent Colleague? CSCW.

[11] LJUNGBLAD, S., NYLANDER, S. \& NØRGAARD, M. (2011) Beyond Speculative Ethics in HRI? Ethical Considerations and the Relation to Empirical Data. HRI.

[12] PARKS, J. A. (2010) Lifting the Burden of Women's Care Work: Should robots Replace the the "Human Touch"? Hypatia, 25, 100-120.

[13] SCB (1999) Från folkbrist till en åldrande befolkning (In Swedish: From lack of people to an ageing population). SCB.

[14] SHARKEY, A. \& SHARKEY, N. (2010a) Granny and the robots: ethical issues in robot care for the elderly. Ethics and Information Technology.

[15] SHARKEY, N. \& SHARKEY, A. (2010b) The crying shame of robot nannies: An ethical appraisal. Interaction Studies, 11, 161-190.

[16] SHARKEY, N. \& SHARKEY, A. (2012) The Rights and Wrongs about Robot Care. IN LIN, P., ABNEY, K. \& BEKEY, G. A. (Eds.) Robot Ethics. MIT Press.

[17] SPARROW, R. \& SPARROW, L. (2006) In the hands of machines? The future of aged care. Minds and Machines, 16, 141-161.

[18] SUNG, J.-Y., GUO, L., GRINTER, R. E. \& CHRISTENSEN, H. I. (2007) My Roomba is Rambo: Intimate Home Appliances. UbiComp.

[19] SUNG, J., GRINTER, R. E. \& CHRISTENSEN, H. I. (2009) "Pimp My Roomba": Designing for Personalization. CHI. Boston, MA.

[20] WHO (2007) Investing in the health workforce enables stronger health systems. WHO. 